\title{
Loasaceae endémicas del Perú
}

\author{
Eric Rodriguez ${ }^{1}$ y Maximilian Weigend ${ }^{2}$
}

${ }^{1}$ Herbarium Truxillense (HUT), Universidad Nacional de Trujillo, Jr. San Martín 392, Trujillo, Perú.

efrr@chanchan.unitru.edu.pe

${ }^{2}$ Freie Universität Berlin, Institut für Biologie, Systematische Botanik und Pflanzengeographie, Altensteinstrasse 6, D14195, Berlin, Alemania.

weigend@zedat.fu-berlin.de

\section{Resumen}

La familia Loasaceae está representada en el Perú por ocho géneros y alrededor de 112 especies (Brako \& Zarucchi, 1993; Ulloa Ulloa et al., 2004), la mayoría herbáceas. En este trabajo se reconoce y categoriza 59 especies y 10 taxones subespecíficos en cinco géneros como endemismos peruanos. El género Nasa es el más rico en especies. Estos taxones endémicos ocupan principalmente las regiones Mesoandina, Puna Húmeda y Seca y Bosques Muy Húmedos Montanos, desde los 1400 hasta los 4700 m de altitud. Diez de los endemismos reconocidos se encuentran representados dentro del Sistema Nacional de Áreas Naturales Protegidas por el Estado.

Palabras claves: Loasaceae, Nasa, Perú, endemismo, plantas endémicas.

\section{Abstract}

The Loasaceae are represented in Peru by eight genera and around 112 species (Brako \& Zarucchi, 1993; Ulloa Ulloa et al., 2004), mainly herbs. Here we recognize 59 species and 10 infra-specific taxa in five genera as Peruvian endemics. Nasa is the genus with the largest number of endemic species. These endemic taxa are found in the Mesoandean, Humid and Dry Puna, and Very Humid Montane Forest regions, between 1400 and $4700 \mathrm{~m}$ elevation. Ten of these taxa have been found in the Peruvian protected areas system.

Keywords: Loasaceae, Nasa, Peru, endemism, endemic plants.

\section{Caiophora carduifolia C. Presl}

\section{LC}

Publicación: Reliq. Haenk. 2(1): 42. 1831.

Colección tipo: T. Haenke s.n.

Herbarios: PR.

Nombre común: D esconocido.

Registro departamental: AP, AR, CU, HV, JU, LI, SM.

Regiones Ecológicas: MA, PSH; 2879-4150 m.

SINANPE: Sin registro.

Herbarios peruanos: CUZ (7), HUT (1), USM (11).

Observaciones: Bejuco. La especie en sentido más amplio se distribuye desde San Martín hasta Cusco y comprende actualmente varios taxones mal definidos. Al parecer pertenece a un complejo de hasta siete especies distintas, todavía sin resolver. Siempre ha sido confundida con Caiophora cirsiifolia. La forma típica proviene del centro del Perú. En Brako \& Zarucchi (1993) se menciona que sólo es conocida de Huánuco; sin embargo, ahora conocemos que el tipo de Haenke s.n. mencionado por ellos proviene de Junín. Es una especie trepadora, con su centro de distribución al norte y al sur de Junín, llegando hasta Lima al oeste; poblaciones aparentemente en buen estado de conservación han sido vistas en estas localidades.

\section{Caiophora cirsiifolia C. Presl}

\section{LC}

Publicación: Arnaldoa 10(1): 80, f. 1B-D, 2A. 2003.

Colección tipo: T. Haenke s.n.

Hemarios: PR.

Nombre común: Ortiga colorada, ortiga macho, pucashinua.

Registro departamental: AN, AP, AR, AY, CA, HV, LI, MO, TA.

Regiones Ecológicas: MA, PSH; 2100-4000 m.

SINAN PE: PNH

Herbarios peruanos: CUZ (1), HAO (2), HUSA (3),

HUT (12), MOL (3), USM (20).
Observaciones: Bejuco, aparentemente restringido a la vertiente occidental y valles interandinos del centro del país. Presenta varias formas regionales; las consideradas aquí están referidas a la forma típica de Huarochirí (Lima). Se considera que existen hasta cuatro formas distintas tanto en el sur como en el norte del Perú. Se estima que está bien representada en el país. Se conocen numerosas poblaciones en Chiquián (Ancash), en Infiernillo (Lima), en Huaytará(Huancavelica), camino Nazca-Puquio (Ayacucho) y cerca de Tarata (Tacna). En algunos poblados, individuos son utilizados con propósitos medicinales.

\section{Caiophora grandiflora (G. Don) Weigend \& M. Ack.} LC

Publicación: Arnaldoa 10(1): 84, f. 3 E-F. 2003.

Colección tipo: Fl. Peruv. Lam. 447.

Hembarios: sd.

Nombre común: Urtica.

Registro departamental: AN, CA, HU, JU.

Regiones Ecológicas: MA, PSH, AA; 2800-4400 m.

SINANPE: PNH

Herbarios peruanos: CPUN (1), HAO (1), HUT (6), USM (8).

Observaciones: Bejuco conocido de numerosas poblaciones, la mayoría proveniente de Ancash. Forma poblaciones grandes. Esta especie se reconoce aquí en el sentido más amplio; otras formas regionales se hallan básicamente en Junín. Abundante en gran parte de su distribución. Relacionada con Caiophora ontorta de Ecuador y Piura (Perú), e incluida por Brako \& Zarucchi (1993) en sinonimia.

Nota del Editor: En la versión on line de este artículo han sido omitidos los mapas del Perú que ilustraban el Registro departamental. Para ubicar las abreviaturas de los departamentos vea al final del artículo. 


\section{Caiophora macrantha Killip}

\section{VU, Bla}

Publicación: J. Wash. Acad. Sci. 18: 94. 1928.

Colección tipo: J.F. Macbride 4468

Herbarios: US.

Nombre común: D esconocido.

Registro departamental: HU, JU, PA.

Regiones Ecológicas: MA, PSH; 3600$4000 \mathrm{~m}$.

SINANPE: Sin registro.

Hemarios penuanos: HUT (1), USM (3).

Observaciones: Bejuco conocido de varias localidades en un área reducida en el centro del país. Esta especie fue originalmente recolectada por Macbride en 1923. Especie muy afín a Caiophora cirsiifolia, con la cual falta aclarar sus límites en una revisión taxonómica.

\section{Caiophora madrequisa Killip}

\section{VU, Blab(iii)}

Publicación: J. Wash. Acad. Sci. 18: 94. 1928.

Colección tipo: O.F. Cook \& G.B. Gilbert 294

Herbarios: F, US.

Nombre común: D esconocido.

Registro departamental: $\mathrm{AP}, \mathrm{AY}, \mathrm{CU}, \mathrm{JU}$, PU.

Regiones Ecológicas: MA, PSH; 1800$4150 \mathrm{~m}$.

SINANPE: SNA

Herbarios peruanos: CUZ (4), HUT (1), MOL (1), USM (7).

Observaciones: Planta trepadora leñosa conocida de poblaciones fragmentadas en bosques de neblina. Es una especie variable que comprende varias formas regionales. Abundante en localidades intervenidas, pero se halla en una sola área protegida y en los límites del Parque Nacional Manu.

\section{Caiophora peduncularis (C. Presl) Weigend \& M. Ack.}

$$
\text { VU, D2 }
$$

Publicación: Arnaldoa 10(1): 78, f. 1A, 2 B-C. 2003.

Colección tipo: M. Weigend et al. 97/ 465

Herbarios: F, M; USM!.

Nombre común: Desconocido.

Registro departamental: JU, LI.

Regiones Ecológicas: MA, PSH, PAR; $1500-4200 \mathrm{~m}$.

SINAN PE: Sin registro.

Herbarios peruanos: USM (tipo).

Observaciones: Especie trepadora afín a Caiophora cirsiifolia, aparentemente restringida al Departamento de Lima y a la zona limítrofe con el de Junín. Conocida mayormente de colecciones antiguas desde 25 hasta 125 años. Registros de Cajamarca son dudosos. Las subpoblaciones en la localidad original están formadas por pocos individuos. Mientras no se efectúen otras colecciones de otras poblaciones y se verifique su rango geográfico, será considerada como amenazada.
7. Caiophora ptenosperma (Ruiz \& Pav. ex G. Don) Unb. \& Gilg.

\section{VU, B lab(iii)}

Publicación: Nova Acta Acad. Caes. Leop.Carol. German. Nat. Cur. 76: 324. 1900.

Colección tipo: Fl. Peruv. Lam. 448.

Herbarios: MA.

Nombre común: D esconocido.

Registro departamental: JU.

Regiones Ecológicas: MA, PSH; 2200$3700 \mathrm{~m}$.

SINANPE: Sin registro.

Herbarios penuanos: HUT (1), USM (1).

Observaciones: Este nombre representa un complejo de por lo menos tres especies más; aquí se trata en sentido restringido que incluye solamente las poblaciones de Junín. Esta especie trepadora es localmente abundante, pero ha sido escasamente recolectada probablemente por las flores verdes que se confunden con el follaje de la vegetación. Considerada por Brako \& Zarucchi (1993) como no endémica. Por su distribución limitada se considera amenazada.

\section{Caiophora stenocarpa Urb. \& Gilg}

$$
\text { EN, Bla }
$$

Publicación: Nova Acta Acad. Caes. Leop.Carol. German. Nat. Cur. 76: 296. 1900.

Colección tipo: J. Maclean s.n.

Herbarios: K.

Nombre común: D esconocido.

Registro departamental: CU, HV.

Regiones Ecológicas: MA; 2800-3000 $\mathrm{m}$.

SINAN PE: Sin registro.

Herbarios peruanos: CUZ (1), HUSA (1), USM (2).

Observaciones: Esta es una especie aceptada en sentido amplio para incluir las poblaciones de Cusco. Planta trepadora cuyas flores verdosas no son conspicuas y por ello se presume que no ha sido recolectada adecuadamente. Las poblaciones en Cusco son abundantes, pero no las de la localidad original.

\section{Caiophora tenuis Killip}

\section{DD}

Publicación: J. Wash. Acad. Sci. 18: 93. 1928.

Colección tipo: J.F. Macbride 3560

Herbarios: F, US.

Nombre común: D esconocido.

Registro departamental: AN, HU.

Regiones Ecológicas: MA, PSH; 2200$3900 \mathrm{~m}$.

SINANPE: Sin registro.

Hemarios peruanos: HUT (3), USM (4).

Observaciones: Esta es una especie de dudosa taxonomía. Es afín a Caiophora carduifolia. Planta trepadora que tiene morfología foliar variable, lo mismo que las flores, que varían en coloración, si bien la forma típica las presenta amarillas. No se la categoriza hasta aclarar su estatus taxonómico. 


\section{Caiophora vargasii Standl. \& F.A. Barkley}

$$
\text { EN, B1ab(iii) }
$$

Publicación: Bull. Torrey Bot. Club 74(1): 81. 1947.

Colección tipo: C. Vargas C. 2392

Hemarios: F.

Nombre común: D esconocido.

Registro departamental: CU.

Regiones Ecológicas: MA; 2800-3800 m.

SINANPE: Sin registro.

Herbarios penuanos: CUZ (1), HUSA (1), USM (1)

Observaciones: Esta planta escandente se conoce del sur del país de poblaciones pequeñas. Estas poblaciones ocupan ambientes modificados por el ser humano. Presenta afinidades con Caiophora canarinoides de Puno y Bolivia.

\section{Mentzelia grandiflora G. Don \\ CR, B lab(iii)}

Publicación: Gen. Syst. 4: 66. 1834.

Colección tipo: H. Ruiz \& J. Pavón s.n.

Herbarios: MA

Nombre común: D esconocido.

Registro departamental: AR, LI.

Regiones Ecológicas: D ST; $60-600 \mathrm{~m}$.

SINAN PE: RNL

Herbarios peruanos: USM (6).

Observaciones: Esta entidad biológica de plantas subarbustivas se acepta provisionalmente en el rango de especie. Tiene afinidades muy cercanas con M entzelia scabra. Está restringida a unas pocas zonas de vegetación de lomas, en donde es rara. Se la considera en peligro crítico debido a que posiblemente sea extinta en varias localidades del D epartamento de Lima, las que siendo naturalmente fragmentadas están amenazadas por la expansión urbana y agropecuaria.

\section{Mentzelia heterosepala Weigend \& E. Rodr.}

\section{LC}

Publicación: Arnaldoa 5(1): 52, 55, 56, f. 1. 1998.

Colección tipo: E. Rodríguez 1298 a

Hembarios: M, MO ; HAO!, HUT!, USM!.

Nombre común: D esconocido.

Registro departamental: AM, AN, CA, LL.

Regiones Ecológicas: BS; 1100-1760 m. SINAN PE: Sin registro.

Herbarios peruanos: $\mathrm{HAO}$ (isotipo+3), HUT(isotipo+5), USM (isotipo+3).

Observaciones: Planta arbustiva aparentemente restringida a los valles secos de la cuenca del río Marañón. Se ha observado numerosas subpoblaciones grandes, de varios individuos, en el valle del Marañón aisladas unas de otras. Su distribución en hábitats secos sin la intervención de la mano del hombre hacen que esta especie no se la considere amenazada y sea considerada de preocupación menor.
13. Nasa aspiazui (J.F. Macbr.) Weigend

$$
\text { EN, Blab(iii) }
$$

Publicación: Rev. Per. Biol. (en prensa) 2006.

Colección tipo: A. Weberbauer 6669

Herbarios: F, US; MOL!, USM!.

Nombre común: D esconocido.

Registro departamental: JU.

Regiones Ecológicas: $\mathrm{BPM}, \mathrm{BMHM}$; 3200-3300 m.

SINAN PE: Sin registro.

Henbarios penuanos: MOL (isotipo), USM (isotipo).

Observaciones: Especie herbácea conocida solamente del lado oriental del D epartamento de Junín. El tipo fue recolectado hace más de 90 años por Weberbauer, y en 1978 otro ejemplar adicional fue recolectado en Concepción. No se conoce el estado actual de sus poblaciones; sin embargo, por existir en un hábitat rápidamente reducido por la acción del hombre está probablemente en peligro.

\section{Nasa callacallensis Weigend \& E. Rodr.}

$$
\text { EN, B 1ab(iii)+2a }
$$

Publicación: Rev. Per. Biol. (en prensa) 2006.

Colección tipo: J.J. Wurdack 1753

Hemarios: F, NY, UC, US; USM!.

Nombre común: Ishanga.

Registro departamental: AM.

Regiones Ecológicas: BPM; 3000-3200 m. SINANPE: Sin registro.

Herbarios penuanos: HAO (1), HUT (1), USM (holotipo+1).

Observaciones: Arbusto, conocido solamente de dos localidades cercanas en Amazonas. La extensión de la presencia se estima en $2000 \mathrm{~km}^{2}$. Consecuencia de la fuerte colonización en la zona, ha sido afectada la población original; sin embargo, se ha registrado poblaciones fragmentadas en los últimos bosques de Polylepis y Weinmannia arriba del sitio arqueológico de Kuelap y en la misma cordillera de la colección tipo. Existe un número reducido de poblaciones fragmentadas constituidas de pocos individuos y por no encontrarse en áreas protegidas se considera muy amenazada.

\section{Nasa carnea (Urb. \& Gilg) Weigend}

$$
\text { EN, B 1ab(iii)+2a }
$$

Publicación: Rev. Per. Biol. (en prensa) 2006.

Colección tipo: A. Weberbauer 4082

Henbarios: $B(d), G$.

Nombre común: Ortiga.

Registro departamental: CA.

Regiones Ecológicas: MA; 2000-3000 m.

SINAN PE: Sin registro.

Herbarios penuanos: $\mathrm{CPUN}(4), \mathrm{HAO}(1)$, HUT (1), USM(2).

Observaciones: Esta especie herbácea se conoce de poblaciones pequeñas y probablemente efímeras. El material examinado es ligeramente heterogéneo; es necesario realizar mayores colecciones y observaciones en campo. Weigend (1999) menciona que los especímenes de Lambayeque (Incahuasi) son morfológicamente algo diferentes a las colecciones de Cajamarca (Chota), de donde procede el tipo; esos ejemplares han sido reconocidos en una especie distinta, $\mathrm{N}$ asa lambayequensis. 


\section{Nasa carunculata (Urb. \& Gilg) Weigend}

$$
\text { VU, B1ab(iii) }
$$

Publicación: Rev. Per. Biol. (en prensa) 2006.

Colección tipo: W. Lobb 358

Herbarios: K.

Nombre común: D esconocido.

Registro departamental: AN, AY, HV.

Regiones Ecológicas: PSH; 2400-3600

$\mathrm{m}$.

SINANPE: Sin registro.

Herbarios peruanos: HUSA (1).

Observaciones: Esta planta arbustiva se conoce de varias poblaciones en el centro del país. Como L oasa carnea, no fue incluida por Brako \& Zarucchi (1993) como un endemismo. Las poblaciones en Ayacucho fueron redescubiertas en el 2000, luego de 74 años. Las poblaciones de esta especie son en muchos casos inaccesibles y posiblemente sean más abundantes.

\section{Nasa chenopodiifolia (Juss.) Weigend}

\section{LC}

Publicación: Rev. Per. Biol. (en prensa) 2006.

Colección tipo: J. Jussieu s.n.

Herbarios: F, P.

Nombre común: D esconocido.

Registro departamental: AM, AN, AR, LL, LI, MO.

Regiones Ecológicas: D ST, MA; 360$3400 \mathrm{~m}$.

SINANPE: Sin registro.

Herbarios penuanos: CUZ (1) HUSA (1), HUT(8), USM (16).

Observaciones: Esta es una hierba conocida de numerosas localidades dispersas. Presenta variabilidad en las características de su hábitat, especialmente de suelos y humedad. En el pasado esto sirvió para reconocer otros taxones, pero Weigend (1998) aclaró que estas forman una especie natural. Localmente forma poblaciones grandes.

\section{Nasa colanii D ostert \& Weigend}

$$
\text { EN, Bla }
$$

Publicación: Harvard Pap. Bot. 4(2): 461. 1999.

Colección tipo: P.J. Barbour 3573

Herbarios: MO; USM!

Nombre común: D esconocido.

Registro departamental: AM.

Regiones Ecológicas: BPM, BMHM; 3000 m.

SINANPE: ZRCC

Herbarios peruanos: USM (isotipo).

Observaciones: Esta hierba se conoce solamente de dos poblaciones en laZona Reservada Cordillera Colán. Aparentemente no ha vuelto a ser recolectada desde 1978. O bservaciones recientes indican que el área en esta cordillera se encuentra inalterada y la carencia de colecciones recientes se debe a la falta de exploración botánica.
19. Nasa contumazensis Weigend \& E. Rodr.

NT

Publicación: Bot. Jahrb. Syst. 124(4): 373, 375, f. 16. 2003.

Colección tipo: A. Sagástegui A. et al. 9755

Herbarios: M, MO, NY; HUT, USM.

Nombre común: Ishguin, ortiga.

Registro departamental: CA.

Regiones Ecológicas: MA; $1800-2680$

$\mathrm{m}$.

SINAN PE: Sin registro.

Herbarios peruanos: HAO (2), HUT (holotipo+7), USM (2).

Observaciones: Esta hierba, de ambientes secundarios, ha sido recolectada de la vertiente Pacífica de Cajamarca. Se conoce de un área pequeña menor a los $500 \mathrm{~km}^{2}$, en la provincia de Contumazá. Por el hábitat que ocupa se considera que está poco afectada por las actividades humanas.

\section{Nasa cymbopetala (Urb. \& Gilg) Weigend subsp. cymbopetala}

\section{LC}

Publicación:

Colección tipo: A. Weberbauer 2758

Herbarios: B, F.

Nombre común: Ortiga, puca shinua.

Registro departamental: AN, JU, LI.

Regiones Ecológicas: MA, PSH; 3000$4350 \mathrm{~m}$.

SINANPE: PNH

Herbarios penuanos: CPUN (2), HUT(6), MOL (2), USM (6).

Observaciones: Esta planta herbácea se conoce de numerosas localidades en el centro occidente del país. Las localidades conocidas en Ancash provienen de la Cordillera Blanca y las de Lima de la cuenca del río Rímac, en donde las poblaciones son grandes.

\section{Nasa dillonii Weigend}

\section{CR, B lab(iii)}

Publicación: Nordic J. Bot. 20(1): 18, f. 2. 2000.

Colección tipo: M.O. Dillon et al. 4416

Henbarios: F, MO, US.

Nombre común: D esconocido.

Registro departamental: CA.

Regiones Ecológicas: MA; 1200-2000

m.

SINAN PE: Sin registro.

Herbarios penuanos: HUT (1), USM (1).

Observaciones: Esta especie herbácea se conoce solamente del bosque de Monteseco (Santa Cruz) y probablemente habitaba en los fragmentos boscosos en los alrededores de ese bosque, en la vertiente occidental. Por ser una especie típica de bosque montano está en peligro de desaparecer a la par que éstos están siendo deforestados. 


\section{Nasa driesslei Weigend}

$$
\text { VU, B lab(iii) }
$$

Publicación: Nordic J. Bot. 20(1): 20-22, f. 32000.

Colección tipo: M. Weigend et al. 97/ 400

Herbarios: F, M; HUT, USM.

Nombre común: D esconocido.

Registro departamental: AM.

Regiones Ecológicas: $\mathrm{BPM}, \mathrm{BMHM}$; 3000-3100 m.

SINAN PE: Sin registro.

Herbarios peruanos: HAO (1), HUT

(isotipo+1), USM (isotipo+3).

Observaciones: Esta especie de plantas herbáceas se conoce de un área reducida en la cuenca alta del Utcubamba. Las poblaciones están formadas por poco individuos. Está potencialmente amenazada por la ampliación de zonas agrícolas, de pastoreo y turismo (Kuelap); sin embargo, podrían encontrarse poblaciones adicionales en los alrededores del rango de registro.

\section{Nasa dyeri (Urb. \& Gilg) Dostert \& Weigend subsp. australis D ostert \& Weigend}

\section{NT}

Publicación: Harvard Pap. Bot. 4(2): 467, f. 1H, 2F, 3E-F, 9A-E. 1999.

Colección tipo: N. D ostert 98/ 80C

Herbarios: F, K, M, MO, NY, US; $\underline{\mathrm{CPUN}}$,

HAO!, USM!.

Nombre común: D esconocido.

Registro departamental: AM.

Regiones Ecológicas: BMHP; 1000$1900 \mathrm{~m}$.

SINAN PE: Sin registro.

Herbarios penuanos: $\mathrm{HAO}$ (isotipo), USM (isotipo+1).

Observaciones: Hierba que forma parte del sotobosque en la Serranía de Bagua. En esa localidad, este taxón fue recolectado en 1978; desde entonces esa localidad ha sido escasamente herborizada. En 1998, se halló poblaciones en fructificación. Observaciones recientes indican que el área se encuentra inalterada y la carencia de colecciones recientes más bien se debe a la falta de exploración botánica.

\section{Nasa formosissima Weigend}

$$
\text { VU, B lab(iii) }
$$

Publicación: Bot. Jahrb. Syst. 124(4): 368, f. 14. 2003.

Colección tipo: M. Weigend et al. 97/ 377

Herbarios: F, K, M, US; USM!.

Nombre común: Ortiga.

Registro departamental: AM, CA, LL.

Regiones Ecológicas: $\mathrm{MA}, \mathrm{BMHM}$; 2800- $2950 \mathrm{~m}$

SINANPE: Sin registro.

Herbarios peruanos: CPUN (1), HUT

(holotipo+1), USM (isotipo+1).

Observaciones: Hierba anual que se conoce de tres localidades en el norte del país. Localmente es una especie con poblaciones de pocos individuos. O cupa una región biogeográfica transicional entre la del bosque montano y la mesoandina. Probablemente, el rango de distribución de esta especie sea mayor.

\section{Nasa glandulosissima Weigend}

$$
\text { EN, Bla }
$$

Publicación: Rev. Per. Biol. (en prensa) 2006.

Colección tipo: M. Weigend \& N. D ostert $98 / 282$

Hemarios: F, M; CPUN, USM.

Nombre común: D esconocido.

Registro departamental: PI.

Regiones Ecológicas: MDE; 1500- 1800 $\mathrm{m}$.

SINAN PE: Sin registro.

Herbarios peruanos: CPUN (isotipo), USM (isotipo).

Observaciones: Hierba de hojas peltadas, conocida sólo de la localidad original. Localmente abundante, pero rara por no existir colecciones adicionales. Probablemente existan otras poblaciones fuera de su rango conocido. Habita en bosques fragmentados del noroeste, de la vertiente del Pacífico.

\section{Nasa hastata (Killip) Weigend CR, Blab(iii)}

Publicación: Rev. Per. Biol. (en prensa) 2006.

Colección tipo: J.F. Macbride \& W. Featherstone 416

Herbarios: F, US.

Nombre común: D esconocido.

Registro departamental: LI.

Regiones Ecológicas: MA; 2500 m.

SINANPE: Sin registro.

Herbarios peruanos: Ninguno.

Observaciones: Esta especie herbácea es pobremente conocida. No ha vuelto a ser recolectada desde 1922. La localidad original ubicada en la cuenca del Rímac está severamente afectada por deforestación de matorrales y bosques. Ello y la ausencia de colecciones recientes sugieren que esta especie podría estar extinta.

\section{Nasa humboldtiana (Urb. \& Gilg) Weigend subsp. obliqua D ostert \& Weigend \\ CR, A1c; B1a}

Publicación: Harvard Pap. Bot. 4(2): 164, f. 1d. 8h. 1999.

Colección tipo: J. Soukup 3826

Herbarios: US.

Nombre común: D esconocido.

Registro departamental: CA.

Regiones Ecológicas: MA; 1200-1800 m.

SINANPE: Sin registro.

Herbarios peruanos: CPUN (1), HUT (3), USM (2).

Observaciones: Esta hierba anual se conoce de un área reducida, en el suroccidente de Cajamarca, en poblaciones fragmentadas. En los herbarios está representada por dos ejemplares recolectados en la localidad original y alrededores, tras un lapso de más de 50 años. Esta especie está restringida a los bosques de la vertiente occidental de Cajamarca y dado el impacto de la deforestación en ellos se considera este taxón severamente afectado por la pérdida de hábitat. 


\section{Nasa humboldtiana (Urb. \& Gilg) Weigend subsp. tricolorDostert \& Weigend}

\section{CR, A1c; Bla}

Publicación: Harvard Pap. Bot. 4(2): 164, f. 2a, 8 a-d. 1999.

Colección tipo: M. Weigend \& N. D ostert 98/ 186

Herbarios: B, F, K, M, MO, NY, P, S, US,

$\mathrm{W}$; CPUN, HAO !, USM?.

Nombre común: Desconocido.

Registro departamental: PI.

Regiones Ecológicas: MA; 1700- 2500

$\mathrm{m}$.

SINANPE: Sin registro.

Herbarios peruanos: CPUN (isotipo), HAO (isotipo+1), HUT (isotipo), USM (isotipo).

Observaciones: Taxón herbáceo que en la década de 1970, Arnaldo López et al. ubicaron hasta 15 poblaciones en la ruta YacupampaCuyas. Este taxón está en la actualidad restringido a tres subpoblaciones en el bosque de Cuyas y alrededores.

\section{Nasa insignis Weigend \& E. Rodr.}

\section{CR, A1c; Bla}

Publicación: Rev. Per. Biol. (en prensa) 2006.

Colección tipo: S. Leiva et al. 1743

Herbarios: F, M; HAO!

Nombre común: D esconocido.

Registro departamental: CA.

Regiones Ecológicas: MA; 1200-1880

m.

SINANPE: Sin registro.

Herbarios peruanos: $\mathrm{CPUN}(1)$ HAO (isotipo+1), HUT(isotipo+2), $\mathrm{USM}(2)$.

Observaciones: Hierba de hojas peltadas, conocida de la vertiente occidental del sur de Cajamarca. Habita los remanentes de dos bosque de la vertiente occidental, tanto en forma solitaria como en subpoblaciones de muchos individuos. Al igual que otras especies de estos bosques, está en peligro de desaparecer a la par que éstos están siendo deforestados.

\section{Nasa kuelapensis Weigend}

$$
\text { CR, A1c; B1 }
$$

Publicación: Bot. Jahrb. Syst. 124(4): 366, 368, f. 13. 2003

Colección tipo: T. Henning \& C. Schneider 244

Hemanios: BSB; USM!.

Nombre común: D esconocido.

Registro departamental: AM.

Regiones Ecológicas: BPM, BMHM; 3000

$\mathrm{m}$

SINANPE: Sin registro.

Herbarios penuanos: USM (holotipo)

Observaciones: Esta hierba se conoce sólo del ejemplar tipo recolectado en los alrededores del sitio arqueológico de Kuelap. Esta población ocupa ambientes intervenidos. Posiblemente se encuentre fuera del rango actual de recolecta; sin embargo, en la localidad del tipo está siendo amenazada por la remoción del matorral que habita.

\section{Nasa lambayequensis Weigend}

VU, B lab(iii)

Publicación: Novon 14(1): 138-139, f. 3.

2004.

Colección tipo: A. Sagástegui A. et al. 12872

Herbarios: F, MO; HUT!.

Nombre común: D esconocido.

Registro departamental: LA.

Regiones Ecológicas: PAR; $3000 \mathrm{~m}$.

SINAN PE: Sin registro.

Herbarios peruanos: HAO (1), HUT (holotipo+1),

Observaciones: Esta especie arbustiva se conoce solamente de una localidad. Forma poblaciones con pocos individuos bajo las grandes rocas y entre piedras en la jalca. La colección de herbario más reciente fue realizada en el 2000.

\section{Nasa laxa (J.F. Macbr.) Weigend}

$$
\text { VU, Blab(iii) }
$$

Publicación: Rev. Per. Biol. (en prensa) 2006.

Colección tipo: A. Weberbauer 6993

Hemarios: F; MOL!, USM!.

Nombre común: Ishguin.

Registro departamental: CA, LL.

Regiones Ecológicas: MA; 2200- $2650 \mathrm{~m}$.

SINANPE: Sin registro.

Herbarios peruanos: $\mathrm{HAO}(1), \mathrm{HUT}(5)$, MOL (isotipo), USM (isotipo+2).

Observaciones: Esta es una especie herbácea conocida de subpoblaciones pequeñas en la provincia de Contumazá. Esta planta es típica de los bosques de la vertiente occidental que se hallan amenazados por la deforestación. Posiblemente la población de La Libertad esté extinta.

\section{Nasa lenta (J.F. Macbr.) Weigend}

EN, B 1ab(iii)

Publicación: Rev. Per. Biol. (en prensa) 2006. Colección tipo: A. Weberbauer 6722

Hemanios: F; MOL!, USM!.

Nombre común: D esconocido.

Registro departamental: HU, SM.

Regiones Ecológicas: BPM, BMHM; $3100 \mathrm{~m}$.

SINANPE: PNRA

Herbarios peruanos: HUT (1), MOL (isotipo), USM (isotipo+2).

Observaciones: Hierba conocida de tres poblaciones grandes y abundantes de los bosques montanos. Esta especie fue recolectada inicialmente en 1913 y nuevamente, luego de más de 80 años. Posiblemente abunde en lugares poco investigados de la vertiente oriental de los bosques montanos de San Martín a Huánuco.

\section{Nasa limata (J.F. Macbr.) Weigend}

\section{VU, B lab(iii)}

Publicación: Rev. Per. Biol. (en prensa) 2006. Colección tipo: C. Vargas C. 1456

Herbarios: F, G, K, US; CUZ!.

Nombre común: Desconocido.

Registro departamental: AP, AY, CU, PU. Regiones Ecológicas: MA, PSH, BPM, BMHM; 2800- $4250 \mathrm{~m}$.

SINANPE: SNA

Herbarios peruanos: CUZ (isotipo+5), HUSA (2), USM (2). 
Observaciones: Hierba conocida de cinco poblaciones en el centrosur del país. Las colecciones más recientes provienen de fragmentos de bosques nublados de Ayacucho y Apurímac. En estos, las poblaciones son grandes, pero potencialmente amenazados por la actividad antropogénica.

\section{Nasa longivalvis E. Rodr. \& Weigend}

\section{LC}

Publicación: Rev. Per. Biol. (en prensa) 2006.

Colección tipo: E. Rodríguez \& V. Medina 2557

Herbarios: BSB, F, MO; $\underline{\text { HAO, HUT, USM. }}$

Nombre común: Ishanga colorada.

Registro departamental: LL.

Regiones Ecológicas: MA; 2743- 3550 m.

SINANPE: Sin registro.

Herbarios peruanos: HAO (isotipo+2), HUT (holotipo+3), USM (isotipo+3).

Observaciones: Esta hierba se conoce de numerosas subpoblaciones en dos provincias orientales de La Libertad, donde crece mayormente en un hábitat agreste e inhóspito para las actividades humanas. A pesar del área pequeña que ocupa y sin protección por el Estado, se considera que por su hábitat está fuera de peligro.

\section{Nasa macrantha (Urb. \& Gilg) Weigend}

\section{LC}

Publicación: Rev. Per. Biol. (en prensa) 2006.

Colección tipo: A. Weberbauer 2494

Herbarios: B.

Nombre común: D esconocido.

Registro departamental: AP, CU, HU, HV, JU.

Regiones Ecológicas: MA, PSH, AA; 2750-4700 m.

SINAN PE: Sin registro.

Herbarios peruanos: CUZ (3), HUSA (2), USM (9).

Observaciones: Esta especie herbácea se conoce de más de diez localidades del centro y sur del país. Ha sido recientemente recolectada en Apurímac (cuenca del Matará) y Cusco (cuenca del Vilcanota). Esta especie también ha sido reportada en colecciones de herbario de una planta del mercado de plantas medicinales de Junín.

\section{Nasa macrornhiza (Urb. \& Gilg) Weigend}

$$
\text { VU, D2 }
$$

Publicación: Rev. Per. Biol. (en prensa) 2006.

Colección tipo: A. Weberbauer 3080

Hemarios: $\mathrm{B}(\mathrm{d}), \mathrm{G}$; MOL!.

Nombre común: D esconocido.

Registro departamental: AN.

Regiones Ecológicas: PSH, AA; 4100$4300 \mathrm{~m}$.

SINAN PE: Sin registro.

Herbarios penuanos: HUSA (1), HUT (1), MOL (isotipo), USM (2).
Observaciones: Planta herbácea que, hasta hace poco, sólo era conocida de la colección tipo efectuada por Weberbauer en la Cordillera Negra (Ancash); sin embargo, en 1997 y 2000 se efectuaron dos colecciones cerca de la localidad original. Esta especie está restringida a un área reducida de la Cordillera Negra, pero su rango probablemente se extienda a las partes altas.

\section{Nasa macrothyrsa (Urb. \& Gilg) Weigend}

\section{VU, Bla}

Publicación: Rev. Per. Biol. (en prensa) 2006.

Colección tipo: A. Weberbauer 3907

Herbarios: $F$.

Nombre común: O rtiga macho.

Registro departamental: CA.

Regiones Ecológicas: MA; 2300-3080 m.

SINAN PE: Sin registro.

Herbarios penuanos: CPUN (4), HAO (3); HUT (9); USM (5).

Observaciones: Arbusto restringido a ocho localidades, en un área pequeña en el suroccidente de Cajamarca. Probablemente se encuentre en el norte de La Libertad.

\section{Nasa magnifica (Urb. \& Gilg) Weigend}

LC

Publicación: Rev. Per. Biol. (en prensa) 2006.

Colección tipo: J. Maclean s.n.

Herbarios: K.

Nombre común: D esconocido.

Registro departamental: AN, HV, LI, MO. Regiones Ecológicas: MA, PSH; 2200$3965 \mathrm{~m}$.

SINAN PE: Sin registro.

Herbarios penuanos: HUT (1), USM (12).

Observaciones: Hierba bianual robusta, conocida de más de diez localidades en el centro del país. Presenta poblaciones generalmente grandes y estables, en algunos casos reportadas en el mismo sitio desde hace décadas. Aparentemente, las poblaciones más septentrionales no se encuentran en peligro, pero hacia el sur de su rango las poblaciones pequeñas podrían ser vulnerables.

\section{Nasa moroensis Weigend}

\section{VU, B lab(iii)}

Publicación: Bot. Jahrb. Syst. 124(4): 370-373, f. 15. 2003.

Colección tipo: M. Weigend \& E. Salas 2000/ 601

Hembanios: F, M, NY; HUT, USM!.

Nombre común: D esconocido.

Registro departamental: AN.

Regiones Ecológicas: MDE; 1180- 1600 m.

SINAN PE: Sin registro.

Herbarios peruanos: HUT (isotipo+2), USM (holotipo+2).

Observaciones: Esta especie herbácea se conoce de dos localidades recolectadas en la vertiente del Pacífico de Ancash. Está relacionada con $\mathrm{N}$ asa hastata, otra especie endémica. Esta especie es muy abundante en años húmedosy completamente ausente en años secos, tal vez por ello sería susceptible a cambios climáticos. 


\section{Nasa nubicolorum Weigend}

$$
\text { VU, B1ab(iii) }
$$

Publicación: Nordic J. Bot. 20(1): 22, f. 5. 2000.

Colección tipo: V. Q uipuscoa et al. 1238

Hemarios: M; HAO!, HUT!.

Nombre común: Shanga.

Registro departamental: AM.

Regiones Ecológicas: BMHM; 2500$2700 \mathrm{~m}$.

SINAN PE: Sin registro.

Herbarios peruanos: HAO (isotipo+2), HUT (isotipo+4), USM (1).

Observaciones: Este subarbusto se conoce de ocho subpoblaciones en los alrededores de la localidad original (Laguna de los Cóndores). Su área de distribución es menor a los 100 km², encontrándose la cobertura de bosques reducida por deforestación. No se conoce su estado en otras áreas aledañas, pero no se descarta su distribución en otros bosques montanos.

\section{Nasa otuzcensis Weigend \& E. Rodr.}

$$
\text { EN, Blab(iii) }
$$

Publicación: Bot. Jarhb. Syst. 124(4): 375, 377, f. 17. 2003.

Colección tipo: E. Rodríguez \& A. Angeludis C. 2374

Herbarios: M, MO, NY; HAO!, HUT!,

USM!.

Nombre común: Ortiga.

Registro departamental: LL.

Regiones Ecológicas: MA; 2250-2600

$\mathrm{m}$

SINANPE: Sin registro.

Herbarios peruanos: HAO (isotipo+2), HUT (holotipo+2), USM (isotipo+2).

Observaciones: Hierba anual, aparentemente restringida a la provincia de Otuzco. Esta especie se conoce de más de 10 subpoblaciones conformadas por pocos individuos que florecen y fructifican entre mayo yjulio. El hábitat de esta especie está afectado por severa deforestación y fragmentación.

\section{Nasa pascoensis Weigend}

\section{CR, B1ab(iii)}

Publicación: Nordic J. Bot. 20(1): 25, f. 6. 2000.

Colección tipo: R.B. Foster \& D.N. Smith 9073

Herbarios: F, M, MO; USM!.

Nombre común: D esconocido.

Registro departamental: JU, PA.

Regiones Ecológicas: BMHM; 2300$2500 \mathrm{~m}$.

SINAN PE: PNYC

Herbarios penuanos: USM (isotipo).

Observaciones: Hierba, conocida solamente de dos localidades en el centro del país. Esta especie fue recolectada por primera vez en Junín en 1957 y luego en Pasco en 1982, esta última en el Parque Nacional Yanachaga-Chemillén. En 1997, se intentó ubicar poblaciones de esta especie en ambas localidades, pero sin éxito. Probablemente la actividad agrícola y deforestación han reducido estas subpoblaciones.
44. Nasa picta (H ook. f.) Weigend subsp. picta

\section{LC}

Publicación:

Colección tipo: A. Mathews s.n.

Herbarios: BM, CBG E, G, OXF.

Nombre común: Ishguin, ishguin de león, ortiga, ortiga de burro.

Registro departamental: AM, AN, CA, LL, PI.

Regiones Ecológicas: MA; $1800-3500$ m.

SINAN PE: Sin registro.

Herbarios penuanos: CPUN (4), HAO (6), HUT (25), USM (9).

Observaciones: Planta herbácea de morfología heterogénea conocida de numerosas localidades en el norte del país, ninguna en áreas protegidas. Las subpoblaciones están formadas por numerosos individuos; este carácter y su amplia distribución permiten categorizarla como no amenazada.

45. Nasa picta (H ook. f.) Weigend subsp. pamparomasii Weigend \& E. Rodr.

$$
\text { VU, Bla }
$$

Publicación: Rev. Per. Biol. (en prensa) 2006.

Colección tipo: M. Weigend \& E. Salas 2000/ 619

Herbarios: F, M, NY; HUT, USM.

Nombre común: D esconocido.

Registro departamental: AN.

Regiones Ecológicas: BMHM; 2850$2870 \mathrm{~m}$.

SINAN PE: Sin registro.

Herbarios peruanos: CPUN (1), HUT (isotipo+1), USM (holotipo+2).

Observaciones: Hierba anual que desarrolla al fin de la época de lluvias. Se conoce de dos poblaciones, aisladas entre ellas, ambas en la vertiente occidental de la Cordillera Negra. Estas poblaciones están formadas por poco individuos. Probablemente su rango sea mayor al conocido, pero sus características biológicas dificultan su recolecta.

\section{Nasa poissoniana (Um. \& Gilg) Weigend}

\section{VU, B lab(iii)}

Publicación: Rev. Per. Biol. (en prensa) 2006.

Colección tipo: C. Gay 1975

Herbarios: P.

Nombre común: Desconocido.

Registro departamental: CU.

Regiones Ecológicas: MA; 2900-3800

m.

SINAN PE: Sin registro.

Herbarios peruanos: CUZ (3), HUSA (3), HUT (1), USM (8).

Observaciones: Esta especie herbácea se conoce solamente de un área pequeña, en el centro del D epartamento de Cusco. La mayoría de las colecciones provienen de la cuenca del Vilcanota, de subpobaciones fragmentadas. 


\section{Nasa pongalamesa Weigend}

$$
\text { VU, Blab(iii) }
$$

Publicación: Novon 14(1): 140-141, f. 1-2, 4. 2004

Colección tipo: M. Weigend et al. 2000/ $752 \mathrm{C}$

Hemarios: BM, BSB, M, MO, NY, W; HUT,

USM.

Nombre común: D esconocido.

Registro departamental: CA.

Regiones Ecológicas: PAR; 3500-3600

$\mathrm{m}$.

SINANPE: Sin registro.

Herbarios peruanos: HUT (isotipo+1), USM (holotipo+1).

Observaciones: Arbusto que se conoce solamente de una localidad, en donde tres subpoblaciones fueron halladas en fructificación. Esta especie probablemente se encuentre en lugares aledaños de similar hábitat. Los ambientes paramunos en donde podría habitar esta especie están seriamente deteriorados por pastoreo intensivo, tala de arbustos en los matorrales e incendios intencionales.

\section{Nasa pteridophylla Weigend \& Dostert subsp.} geniculata Weigend \& Dostert

\section{CR, A1c, Bla}

Publicación: Harvard Pap. Bot. 4(2): 459, f. 1e, 7a-d. 1999.

Colección tipo: J. Santisteban \& J. G uevara 079

Herbarios: F, HUT; $\underline{\text { HUT. }}$

Nombre común: D esconocido.

Registro departamental: CA.

Regiones Ecológicas: MA; 1500-1800

m.

SINAN PE: Sin registro.

Herbarios peruanos: CPUN (1), HAO (isotipo), HUT (1), USM (isotipo+2).

Observaciones: Esta especie herbácea es típica de bosques relictos de la vertiente occidental. Se conoce sólo de colecciones efectuadas en Monteseco (Santa Cruz, Cajamarca); sin embargo no se descarta su presencia en otros fragmentos de bosque aledaños. Su estado crítico es evidente por la constante deforestación en esta zona no protegida.

\section{Nasa pteridophylla Weigend \& Dostert subsp. pteridophylla}

\section{LC}

Publicación:

Colección tipo: M. Weigend et al. 97/ 307

Hembarios: $\mathrm{F}, \mathrm{K}, \mathrm{M}, \mathrm{MO}, \mathrm{NY}, \mathrm{W}$; $\underline{\mathrm{HAO}}$

USM.

Nombre común: D esconocido.

Registro departamental: CA, LL.

Regiones Ecológicas: MA; $1400-2600$ $\mathrm{m}$.

SINANPE: Sin registro.

Herbarios peruanos: HAO (isotipo citado), HUT (isotipo), USM (isotipo citado).

Observaciones: Esta planta herbácea se conoce de un área limitada, desde el sur de Cajamarca hasta el norte de La Libertad. Las poblaciones conocidas son grandes y su hábitat no evidencia problemas mayores.
50. Nasa raimondii (Standl. \& Barklay) Weigend

$$
\text { EN, Bla }
$$

Publicación: Rev. Per. Biol. (en prensa) 2006.

Colección tipo: C. Vargas C. 2672

Herbarios: F, UC; $\underline{\text { CUZ. }}$

Nombre común: Angel tauna.

Registro departamental: AP, CU.

Regiones Ecológicas: MA, PSH; 2300$3500 \mathrm{~m}$.

SINANPE: Sin registro.

Herbarios penuanos: HUSA (2), USM (2).

Observaciones: Esta especie herbácea se conoce del sur del país, de dos localidades en un área menor a los $1000 \mathrm{~km}^{2}$. Ha sido recolectada en 2000 de Apurímac, pero raras veces en Cusco, de donde proviene la colección original. Las subpoblaciones están integradas por pocos individuos dispersos, los que son aparentemente desplazados por otras plantas silvestres.

\section{Nasa ranunculifolia (Kunth) Weigend}

\section{NT}

Publicación: Rev. Per. Biol. (en prensa) 2006.

Colección tipo: A. Humboldt \& A. Bonpland s.n.

Herbarios: $\mathrm{P}$

Nombre común: Ortiga de león.

Registro departamental: CA, LL.

Regiones Ecológicas: MA, PAR; 2300$3500 \mathrm{~m}$.

SINANPE: Sin registro.

Herbarios peruanos: CPUN (2), HAO (5), HUT (9), USM (8).

Observaciones: Esta hierba se conoce de numerosas localidades en la parte paramunay mesoandina, del occidente y sur de Cajamarca al norte de La Libertad. Las subpoblaciones presentan numerosos individuos.

\section{Nasa rugosa (Killip) Weigend \\ CR, Blab(iii)}

Publicación: Rev. Per. Biol. (en prensa) 2006.

Colección tipo: J.F. Macbride 4350

Herbarios: F, US.

Nombre común: D esconocido.

Registro departamental: HU.

Regiones Ecológicas: MA; 4000 m.

SINAN PE: Sin registro.

Henbarios peruanos: Ninguno.

Observaciones: Esta especie herbácea, que debe su nombre al carácter de las hojas, se conoce de una sola localidad, la que fue citada como de Junín, pero que hoy se reconoce en Huánuco. Poco se sabe de sus requerimientos de hábitat, asumiéndose pajonales entre rocas. A pesar de repetidos intentos por reubicar poblaciones de esta especie, no ha vuelto a ser observada desde 1923. La localidad original y los alrededores presentan los ambientes naturales muy modificados. 


\section{Nasa sagasteguiiWeigend}

$$
\text { CR, Blab(iii) }
$$

Publicación: Nordic J. Bot. 20(1): 26, f. 7. 2000. Colección tipo: A. Sagástegui A. \& J.M. Cabanillas 8571

Herbarios: M, MO; HUT.

Nombre común: Desconocido.

Registro departamental: PI.

Regiones Ecológicas: PAR; $3000 \mathrm{~m}$.

SINAN PE: Sin registro.

Herbarios peruanos: HUT (isotipo).

Observaciones: Esta hierba se conoce de una localidad en la jalca de Piura. No ha vuelto a ser recolectada desde 1976, encontrándose la localidad original sometida a presión por quema y tala. En 1998 se intentó ubicar esta población, pero sin éxito.

\section{Nasa solaria (J.F. Macbr.) Weigend}

$$
\text { EN, Blab(iii) }
$$

Publicación: Rev. Per. Biol. (en prensa) 2006. Colección tipo: J.F. Macbride \& W. Featherstone 577

Herbarios: F, US.

Nombre común: D esconocido.

Registro departamental: LI.

Regiones Ecológicas: MA, PSH; 2900$4000 \mathrm{~m}$.

SINANPE: Sin registro.

Herbarios peruanos: USM (2).

Observaciones: Esta planta herbácea se conoce solamente de la cuenca del nío Rimac. La localidad original ha sido probablemente destruida. O tras poblaciones en la cuenca están ubicadas al menos en uno de los fragmentos de bosque perennifolio. Por su rango geográfico pequeño y la carencia de recolectas actuales hacen presumir que esta especie se encuentre muy amenazada.

\section{Nasa solata (J.F. Macbr.) Weigend}

$$
\text { CR, Blab(iii) }
$$

Publicación: Rev. Per. Biol. (en prensa) 2006. Colección tipo: A. Weberbauer 6408

Herbarios: F, US.

Nombre común: D esconocido.

Registro departamental: PI.

Regiones Ecológicas: MA, PAR; 2300$3100 \mathrm{~m}$.

SINANPE: Sin registro.

Herbarios peruanos: CPUN (1), USM (1).

Observaciones: Esta especie herbácea se conoce de dos localidades en Piura. En el año 1998, se registró una población adicional, después de la del tipo, arriba de Huancabamba(Piura); esta población era muy pequeña y crecía en un sitio muy intervenido.

\section{6. stolonifera Weigend}

\section{VU, Bla}

Publicación: Novon 14(1): 142, f. 5. 2004 Colección tipo: M. Weigend et al. 2000/ 822 Hemarios: BSB, M; HUT, USM.

Nombre común: D esconocido.

Registro departamental: LL

Regiones Ecológicas: MA; 3050- 3280

$\mathrm{m}$.

SINANPE: Sin registro.

Herbarios peruanos: HUT (isotipo+1), USM (holotipo).
Observaciones: Esta hierba se conoce solamente de bosques fragmentados de A lnus acuminata de la cuenca del Marañón. Esta especie es estolonífera y por ello las subpoblaciones son grandes, probablemente formando clones. Fue recolectada por primera vez en 1960 y luego en 2000. Se considera que existan otras poblaciones en la Provincia de Bolívar.

\section{Nasa stuebeliana (Urb. \& Gilg) Weigend}

\section{VU, Blab(iii)}

Publicación: Rev. Per. Biol. (en prensa) 2006.

Colección tipo: A. Mathews s.n.

Henbarios: BM, CBGE, OXF.

Nombre común: D esconocido.

Registro departamental: AM.

Regiones Ecológicas: BMHM; 1900 $2100 \mathrm{~m}$.

SINANPE: Sin registro.

Herbarios peruanos: CPUN (1), USM (1).

Observaciones: Esta hierba se conoce de un área pequeña de la vertiente oriental de la cordillera central, en la Provincia de Chachapoyas. Se considera que es una especie rara, si bien crece en campos de cultivo, pero es removida por sus pelos urticantes. En el valle del Utcubamba, de donde se conoce esta especie, hay una extensa actividad agropecuaria que presionan las poblaciones conocidas. Posiblemente extinta en parte de su rango.

\section{Nasa tingomaniensis (J.F. Macbr.) Weigend}

\section{NT}

Publicación: Rev. Per. Biol. (en prensa) 2006.

Colección tipo: H.E. Stork \& O.B. Horton 9512

Herbarios: $F, K$.

Nombre común: Cocona masha

Registro departamental: $\mathrm{HU}$.

Regiones Ecológicas: $\mathrm{BMHM}, \mathrm{BMHP}$; 650- $1850 \mathrm{~m}$.

SINANPE: PNTM

Herbarios penuanos: HUT (2), USM (7).

Observaciones: Esta especie herbácea se conoce de numerosas colecciones de herbario procedentes, la mayoría de ellas, de los alrededores de Tingo María y todas en la cuenca del río Huallaga. Si bien su distribución geográfica es reducida, esta especie tiende a ser invasora en ambientes modificados por el ser humano, por lo que se considera que podría encontrarse en otras localidades.

\section{Nasa triphylla (Juss.) Weigend subsp. elegans Dostert \& Weigend}

\section{NT}

Publicación: Harvard Pap. Bot. 4(2): 456, f. 1a, 2e, 6. 1999.

Colección tipo: N. D ostert 98/47c

Herbarios: F, K, M, MO, NY, W; $\mathrm{HAO}$ !, USM!.

Nombre común: D esconocido.

Registro departamental: AM, CA, SM.

Regiones Ecológicas: BMHM; 1400 $2700 \mathrm{~m}$.

SINANPE: Sin registro.

Herbarios peruanos: CPUN (2), HAO (isotipo+4), HUT (1), USM (isotipo+3). 
Observaciones: Planta herbácea, conocida de varias localidades en el nororiente del país, aunque poco recolectada. Probablemente su distribución sea mayory dada su plasticidad en requerimientos de hábitat, no se conocen amenazas inmediatas.

\section{Nasa triphylla (Juss.) Weigend subsp. flavipes Weigend \& Dostert}

$$
\text { VU, B lab(iii) }
$$

Publicación: Harvard Pap. Bot. 4(2): 456, f. 1B, 4H, 5A-E. 1999.

Colección tipo: M. Weigend \& N. D ostert $98 / 203$

Hemanios: F, K, M, MO, NY, W; $\underline{\mathrm{CPUN}}$, HAO!, USM.

Nombre común: D esconocido.

Registro departamental: PI

Regiones Ecológicas: MA; $1700-2500$

m.

SINAN PE: Sin registro.

Herbarios peruanos: CPUN (isotipo+2), HAO (isotipo), USM (isotipo+2).

Observaciones: Taxón herbáceo conocido solamente de tres localidades en Piura y restringida a la zona de Huancabamba.

\section{Nasa umbraculifera E. Rodr. \& Weigend}

$$
\text { EN, Blab(iii)+2a }
$$

Publicación: Rev. Per. Biol. (en prensa) 2006.

Colección tipo: V. Quipuscoa et al. 1208

Herbarios: F, M; HAO!, HUT.

Nombre común: Ishanga, shanga, ortiga.

Registro departamental: AM.

Regiones Ecológicas: BPM; 2700-3500 m.

SINANPE: Sin registro.

Herbarios peruanos: $\mathrm{HAO}$ (isotipo), HUT (tipo citado).

Observaciones: Este subarbusto se conoce de cinco subpoblaciones fragmentadas, en los alrededores de la Laguna de los Cóndores en Leimebamba (Amazonas). Rodríguez \& Weigend (1999) y Weigend \& Rodriguez (2002) consideran que aparentemente está restringida a esta zona. El área de la presencia estimada para esta especie es inferior a los $1000 \mathrm{~km}^{2}$. Probablemente se presente en otras zonas boscosas vecinas, pero su hábitat es amenazado por actividades humanas.

\section{Nasa urentivelutina Weigend}

$$
\text { VU, Bla }
$$

Publicación: Nord. J. Botany 20(1): 2931, f. 9. 2000

Colección tipo: M. Weigend et al. 98/ 308

Herbarios: F, M; CPUN, USM.

Nombre común: D esconocido.

Registro departamental: CA.

Regiones Ecológicas: MA; 2900-3000

m.

SINANPE: Sin registro.

Herbarios peruanos: $\mathrm{CPUN}($ isotipo +2$)$ USM(isotipo+2), HUT(1).
Observaciones: Arbusto conocido de una localidad, en la cuenca del río Marañón en poblaciones con pocos individuos. La localidad original cuenta con hábitats poco intervenidos, tal vez por ubicarse en ambientes poco propicios para la agricultura. Su rango de distribución podría incluir otras localidades en esta cuenca.

\section{Nasa usquliensis Weigend}

$$
\text { EN, Blab(iii) }
$$

Publicación: Syst. Bot. 28(4): 780, f. 1c, 2a-d, 4a, b, d, 10. 2003.

Colección tipo: T. Henning \& C. Schneider 307

Herbarios: BSB, M; HUT, USM.

Nombre común: D esconocido.

Registro departamental: LL.

Regiones Ecológicas: MA; 2150-3200 m.

SINANPE: Sin registro.

Herbarios peruanos: HUT (holotipo), USM (isotipo+1).

Observaciones: Esta especie arbustiva se conoce de una localidad, en la vertiente occidental de La Libertad. Ambas localidades están ubicadas entre las cuencas delos ríos Huancay y Moche. Posiblemente otras poblaciones se encuentren habitando los bosques y matorrales fragmentados. Estos ambientes están afectados por la actividad humana.

\section{Nasa vargasii (J.F. Macbr.) Weigend}

$$
\text { VU, Blab(iii) }
$$

Publicación: Rev. Per. Biol. (en prensa) 2006.

Colección tipo: H.E. Stork et al. 10531

Herbarios: UC.

Nombre común: D esconocido.

Registro departamental: AP, CU, HU.

Regiones Ecológicas: MA; 1700-3400 $\mathrm{m}$.

SINAN PE: Sin registro.

Herbarios peruanos: CUZ (7), HUSA (2), USM (4).

Observaciones: Esta planta herbácea se conoce de poblaciones aisladas y pequeñas. Las poblaciones más distantes se hallan separadas por más de $500 \mathrm{~km}$ en línea recta. En los años 2000 y 2001 se ubicaron poblaciones en Huánuco y Cusco.

\section{Nasa victonii Weigend}

\section{VU, Blab(iii)}

Publicación: Novon 14(1): 142, 144145, f. 6. 2004

Colección tipo: V. Q uipuscoa et al. 2480 Hemanios: BSB, F; HAO, HUSA, HUT.

Nombre común: D esconocido.

Registro departamental: SM.

Regiones Ecológicas: $\mathrm{BPM}, \mathrm{BMHM}$; 2000-3200 m.

SINAN PE: Sin registro.

Herbarios peruanos: HAO (isotipo), HUSA (isotipo), HUT (holotipo+1), USM (1).

Observaciones: Este arbusto subescandente, fue recolectado en el año 2000, de una parte de San Martín poco explorada. Weigend (2004) sugiere que la extensión geográfica es mayor. 


\section{Nasa webenbaueri (Um. \& Gilg) Weigend}

\section{LC}

Publicación: Rev. Per. Biol. (en prensa) 2006.

Colección tipo: A. Weberbauer 4183

Hembarios: $B(d), G$.

Nombre común: D esconocido.

Registro departamental: CA, LA, PI.

Regiones Ecológicas: MA, PAR; 2650$3200 \mathrm{~m}$.

SINAN PE: Sin registro.

Herbarios penuanos: CPUN (1), HAO (1), HUT (2), USM (4).

Observaciones: Esta planta arbustiva se conoce de la vertiente occidental, en el norte del país. Es una especie abundante que crece en ambientes naturalmente fragmentados en donde forma poblaciones grandes, con individuos ramificados y rizomas extendidos.

\section{Presliophytum heucheraefolium (Killip) Weigend}

\section{EN, Blab(iii)}

Publicación: Nasa Conq. S. Amer. 219. 1997.

Colección tipo: J.F. Macbride \& W. Featherstone 2543

Herbarios: F, US.

Nombre común: D esconocido.

Registro departamental: AN, LI.

Regiones Ecológicas: D ST, MDE; 400$1141 \mathrm{~m}$

SINAN PE: Sin registro.

Herbarios penuanos: HUT (1), USM (5).

Observaciones: Planta leñosa, conocida originalmente de la cuenca del río Grande, en la vertiente occidental de la Cordillera Negra. Todos los ejemplares de herbario provienen de esa vertiente y la mayoría de ellos de Ancash. Posiblemente su rango de distribución sea algo mayor y existan otras poblaciones en localidades no herborizadas. Las poblaciones conocidas varían en tamaño de año a año y podrían verse afectadas por la expansión urbana (Lima).

\section{Presliophytum incanum (Graham) Weigend}

\section{LC}

Publicación: Nasa Conq. S. Amer. 219. 1997.

Colección tipo: Cruckshanks s.n.

Herbarios: BM, E, K.

Nombre común: D esconocido.

Registro departamental: $\mathrm{AN}, \mathrm{AR}, \mathrm{AY}, \mathrm{CA}$, HV, IC, LL, LI, MO, PI.

Regiones Ecológicas: DST, MDE; $10-$ $2280 \mathrm{~m}$.

SINAN PE: Sin registro.

Herbarios penuanos: CPUN (2), HAO (5), HUT (17), MOL (4), USM (18).

Observaciones: Este arbusto se conoce de numerosas localidades, ubicadas en el centro de la vertiente del Pacífico. Esta especie ocupa diferentes tipos de ambientes, en una gradiente altitudinal que supera los $2000 \mathrm{~m}$. La mayoría de las poblaciones ocupan lugares agrestes por lo que se considera de preocupación menor.

\section{Xylopodia klaprothioides Weigend}

\section{EN, Blab(iii)}

Publicación: Nasa Conq. S. Amer. 215, f. 76. 1997.

Colección tipo: M. Weigend et al. 97/ 450

Herbarios: M; USM.

Nombre común: D esconocido.

Registro departamental: CA.

Regiones Ecológicas: MA; 2400- 2900

m.

SINANPE: Sin registro.

Herbarios peruanos: HUT(3), USM(isotipo+3).

Observaciones: Esta especie leñosa se conoce de dos poblaciones, en la vertiente occidental del sur de Cajamarca. La población original de donde procede el tipo presenta numerosos individuos, siendo las condiciones ambientales propicias para la especie. La otra población conocida del sur de la localidad original, cerca al límite con La Libertad es muy pequeña y en peligro. 


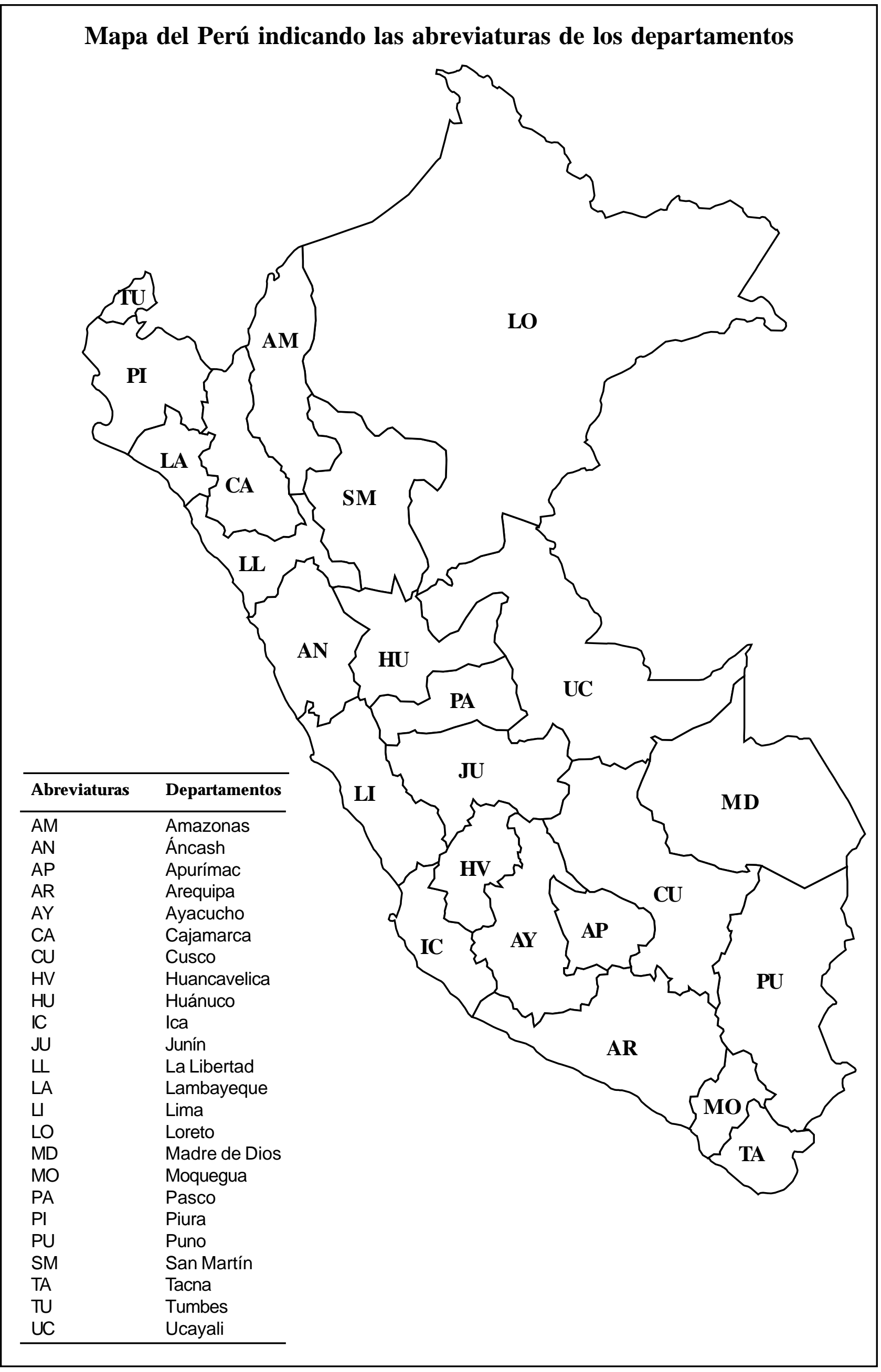

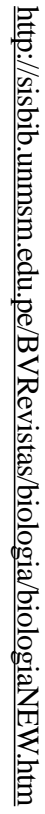

